\title{
МОЖНО ЛИ ИДЕНТИФИЦИРОВАТЬ ВЫСОКОДИФФЕРЕНЦИРОВАННЫЙ РАК УЖЕ ПРИ СОНОГРАФИЧЕСКОМ ИССЛЕДОВАНИИ ЩИТОВИДНОЙ ЖЕЛЕЗЫ?
}

\author{
Бутырский А.Г.', Михайличенко В.Ю. ${ }^{1}$, Дубовенко В.В. ${ }^{2}$, Шупта Ю.Б², Бутырская И.Б.
}

'Медицинская академия имени С.И. Георгиевского ФГАОУ ВО «Крымский федеральный университет (КФУ) имени В.И. Вернадского» МНВО РФ, Симферополь

${ }^{2}$ ООО Клиника Генезис

зГБУз РК РКБ имени Н.А. Семашко

ЦЕЛЬ: оценка диагностической ценности УЗ критериев, которые могут указывать на папиллярный рак щитовидной железы (ПРЩЖ).

МАТЕРИАЛЫ И МЕТОДЫ: проведено обсервационное многоцентровое ретроспективное сплошное исследование среди пациентов, прооперированных по поводу узлов (У) ЩЖ. В исследование вошли взрослые больные без ограничения возраста (18-78 лет) и пола. Всем пациентам амбулаторно проводилось УЗИ ЩЖ, в стационаре выполнялось оперативное вмешательство, объем которого зависел от предоперационных данных и операционных находок. УЗ критериями оценки послужили: умеренная гипоэхогенность, выраженная гипоэхогенность, полициклические/неровные контуры, наличие микрокальцинатов, «выше, чем шире», отсутствие тонкого гипоэхогенного ободка

Объектами исследования послужили 124 медицинские карты стационарных больных с 2014 по 2018 годы. У 32 из них после проводки был выставлен диагноз РЩЖ (у 24 - ПРЩЖ, у 8 - фолликулярный (Ф) РЩЖ) и у 92 - ДУЩЖ. Мода возраста обследованных составила 46 лет, мода среднего размера узла 22 мм.

Данные представлены в процентном формате. Мы сравнивали их методом непараметрической статистики с использованием показателя Пирсона $\left(X n^{2}\right)$ для дискретных переменных; $\mathrm{p}<0,05$ считалось статистически значимым. Для статистического анализа использовали пакет программ MedStat.

PЕЗУЛЬТАТЫ: из исследованных критериев злокачественности все, кроме умеренной гипоэхогенности, достоверно отличались между ДУЗЖ и ПРЩЖ. При сравнении критерий «отсутствие тонкого гипоэхогенного ободка» был сравним с другими критериями по достоверности подозрения на ПРЩЖ с учетом поправки Bonferroni для множественных сравнений.

Чувствительность, специфичность, точность каждого их изучаемых УЗ критериев для ПРщЖ составили соответственно: умеренная гипоэхогенность - 39\%, 60\%, 50\%, выраженная гипоэхогенность - 54\%, 80\%, 67\%, полициклические/неровные контуры - 58\%, 73\%, 66\%, наличие микрокальцинатов - 38\%, 96\%, 67\%, выше, чем шире - 33\%, 89\%, 61\%, отсутствие тонкого гипоэхогенного ободка - 92\%, 33\%, 63\%.

Множественное сравнение с поправкой Bonferroni показало, что среднее количество описанных критериев при ПРЩЖ $(2,87 \pm 1,23)$ статистически отличается от ДУЩЖ $(1,67 \pm 1,08 ;$ р $<0,001)$ и от ФРЩЖ $(1,58 \pm 1,02 ; p=0,027)$, тогда как среднее число для ДУЩЖ и ФРЩЖ сравнимо (p>0,99).

У 2 из 24 больных с ПРЩЖ (9\%) при УЗИ вообще не было выявлено ни одного из упомянутых критериев (1 - с Т1a, 1 - с Т1b); был выставлен диагноз ДУЩЖ. 21 из 24 пациентов (88\%) с ПРЩЖ с двумя и более положительными критериями были оценены правильно, а вот 51 из 92 дУЩЖ (56\%) при УзИ были оценены как ПРЩЖ. Сравнительный анализ суммы положительных критериев показал, что статистически достоверная разница наблюдается при количестве критериев более 2.

ЗАКЛЮЧЕНИЕ Наши исследования показали необходимость применения нескольких сонографических критериев. Точность УЗИ примерно одинакова при наличии от 2 до 4 сонографических критериев; точность УЗИ при наличии 1 или 5 критериев значительно ниже: в первом случае за счет низкой специфичности, а в последнем - за счет редкости одновременного сочетания. Наибольшей точности (74\%) достигает УЗИ при наличии 3 критериев из описанных. Абсолютной точности диагностики ПР ЩЖ при УЗИ на его сегодняшнем уровне достичь не удается.

Невозможно идентифицировать ФР ЩЖ при УЗИ, его сонографическая семиотика не имеет каких-либо специфических симптомов. 